\title{
Molecular Mechanism of Interaction Between Fatty Acid Delta 6 Desaturase and Acyl-CoA by Computational Prediction
}

Jie Cui

Jiangnan University

Haiqin Chen ( $\nabla$ haiqinchen@jiangnan.edu.cn )

Jiangnan University https://orcid.org/0000-0002-2359-2622

Xin Tang

Jiangnan University

Hao Zhang

Jiangnan University

YongQ Chen

Jiangnan University

Wei Chen

Jiangnan University

Original article

Keywords: desaturation, ligand-binding site, molecular docking, pose generation and evaluation, modeling

Posted Date: December 13th, 2021

DOI: https://doi.org/10.21203/rs.3.rs-1147355/v1

License: () (1) This work is licensed under a Creative Commons Attribution 4.0 International License.

Read Full License 


\section{Abstract}

Enzyme catalyzed desaturation of intracellular fatty acids plays an important role in various physiological and pathological processes related to lipids. Limited to the multiple transmembrane domains, it is difficult to obtain their three-dimensional structure of fatty acid desaturases. So how they interact with their substrates is unclear. Here, we predicted the complex of Micromonas pusilla delta 6 desaturase (MpFADS6) with the substrate linoleinyl-CoA (ALA-CoA) by trRosetta software and docking poses by Dock 6 software. The potential enzyme-substrate binding sites were anchored by analysis of the complex. Then, site-directed mutagenesis and activity verification clarified that W290, W224, and F352 were critical residues of the substrate tunnel and directly bonded to ALA-CoA. H94 and H69 were indispensable for transporting electrons with heme. $\mathrm{H} 452$, N445, and H358 significantly influenced the recognition and attraction of MpFADS6 to the substrate. These findings provide new insights and methods to determine the structure, mechanisms and directed transformation of membrane-bound desaturases.

\section{Keypoints}

- The structure of the $\Delta 6$ fatty acid desaturase and substrate complex is modeled.

- The substrate tunnel and key residues of MpFADS6 catalytic activity are determined.

- The new insights to determine the mechanism of the membrane-bound desaturases.

\section{Introduction}

In vivo, membrane proteins are involved in various essential signal transduction, synthesis, and metabolism events, such as the transport of ions and small molecules, and intercellular and intracellular communication. They are also important drug targets. Approximately $60 \%$ of current drugs target membrane proteins (Wang et al. 2020). As a membrane-bound protein, fatty acid desaturase is a necessary component of anabolic long-chain polyunsaturated fatty acids (PUFAs). Numerous studies have illustrated that the ratio of omega-6/omega-3 PUFAs is associated with physiological health (Tosi et al. 2014). Fatty acid delta 6 desaturase (FADS6) is the first step in the metabolic pathway of omega6/omega-3 PUFAs. The activity and preference of FADS6 directly affect the ratio of omega-6/omega-3 PUFAs. It catalyzes the production of linoleic acid (LA) to gamma linolenic acid (GLA), and the production of linoleic acid (ALA) to stearidonic acid (SDA). Among these enzymes, Micromonas pusilla delta 6 desaturase (MPFADS6) reportedly showed a greater preference for ALA (omega-3 PUFA) than LA (omega6 PUFA) (Shi et al. 2015). Molecular analysis of fatty acid desaturases that have significant differences in sequence and substrate specificity in various organisms will promote the understanding of their roles in physiological regulation.

However, the membrane-bound fatty acid desaturases have multiple transmembrane domains that fail to be expressed in Escherichia coli. The hydrophobic surface of fatty acid desaturases hinders detergents from separating and dissolving proteins. These facts have hindered the study of the interactions of 
desaturases with their substrates. The complete lack of structural data has also complicated the sequence-based analysis of the desaturation mechanism. In 2015, the crystal structures of integral membrane stearoyl-CoA desaturase from humans (Wang et al. 2015) and musculus (muSCD1) (Bai et al. 2015)were reported, which advanced investigation of the fatty acid desaturation mechanism. In 2020 , the structure of active muSCD1 was resolved (Shen et al. 2020). However, the crystallization of membranebound desaturases remains an arduous process. Molecular simulation provides a unique method to research the structure of membrane proteins, providing data that complements experimental findings.

The confidence of membrane protein structure prediction increases according to the numbers of membrane protein structures in the database used for derivation and verification (Dowhan et al. 2019; Du et al. 2020; Yang et al. 2020). Compared to soluble protein modeling, approaches for membrane proteins lack tentative structures, which has delayed the development of algorithms and their application. Modeling of membrane proteins mainly focuses on attaining models of unknown proteins without template structures. The mainstream modeling technology mainly comprises homology modeling, folding recognition technology, and $a b$ initio modeling. Homology modeling is suitable for identifying amino acid sequences between query and template higher than $30 \%$. The amino acid sequence identity among the subfamilies of fatty acid desaturases is below this threshold. However, it was recently reported that the root-mean-square deviation (RMSD) of the $\mathrm{G}$ protein-coupled receptor homology model and the actual structure was $2.9 \AA$, and its sequence identity was only $15 \%$ of the template (Chen et al. 2014). Folding recognition technology generates low-resolution protein models for known structures as low as $5-25 \%$. The accuracy of these models rarely reaches an RMSD of 3-4 $\AA$ (Alford et al. 2015). The ab initio approach (Wang and Barth 2015; Yang et al. 2020) is not affected by the template structure, as many membrane-binding proteins have no relevant template for modeling. This can be advantageous or limiting.

Based on the membrane-binding protein model, molecular simulations have been used to constrain and refine the structures (Melcr et al. 2020). In addition to structural refinement, molecular simulation is also applied to determine the ligand-binding sites of proteins, which are the amino acid residues at specific positions of proteins that interact with ligands. The identification of ligand binding sites can further the understanding of the mechanism of molecular interactions and the pathogenesis of diseases, which provides insights for drug discovery and design (Altschul et al. 1990). Wang et al. classified the ligandbinding site detection methods into four categories and discussed their scope of applications (Zhao et al. 2020). It has been reported that the programs LeDock and rDock showed better performance on ligands with fewer than 10 rotatable bonds (Wang et al. 2016). However, the direct action of fatty acid desaturase causes the substrate fatty acyl-CoA to have more than 40 flexible bonds, such as the substrate molecule ALA-CoA of FADS6. It is still important to evaluate and compare the performance of these programs for fatty acid desaturase.

In this study, the muSCD1 crystal structure, the only membrane-bound fatty acid desaturase with activity in the RCSB Protein Data Bank (PDB), was used to evaluate the performance of six modeling methods and docking programs to predicted the complex of MpFADS6 with the substrate linoleinyl-CoA (ALA-CoA). 
The potential binding sites were anchored by analysis for the complex. The contributions of these potential binding residues to the desaturation of MpFADS6 were verified by alanine substitution.

\section{Materials And Methods}

\section{Protein sequence and reference structure}

Amino acid sequence and crystal structure data of muSCD1 were obtained from the PDB (PDB ID: 4YMK). The amino acid sequence of MpFADS6 (Uniport ID: C1MMV2) was obtained from the Uniport. Ligand molecules, heme (PubChem CID: 26945), ferrous ion (PubChem CID: 27284), and stearoyl-CoA (SA-CoA, PubChem CID: 94140) were loaded from the NCBI compound structure library. Substrate linoleinyl-CoA (ALA-CoA) was constructed by Chimera (version 1.13.1) (Forli et al. 2016) based on SA$\mathrm{CoA}$. The ligand was prepared as described by Chimera. Multiple sequence alignments were performed using Clustal Omega (Madeira et al. 2019).

\section{Modeling methods}

In this study, muSCD1 was selected as the target protein to screen the fatty acid desaturase modeling method. The SCD_SW model was generated by the Swiss-model (Waterhouse et al. 2018) (https://swissmodel.expasy.org/interactive) online comparison and manual selection of templates. The SCD_Cl and SCD_CA models were automatically generated by the C-I-TASSER (Yang and Zhang 2015) (https://zhanglab.ccmb.med.umich.edu/C-I-TASSER/) and CATHER suites (Du et al. 2020) (https://yanglab.nankai.edu.cn/CATHER/), respectively. ROBETTA (http://new.robetta.org/), CQUARK (Zheng et al. 2019) (https://zhanglab.ccmb.med.umich.edu/C-QUARK/), and trRosetta (Yang et al. 2020) (https://yanglab.nankai.edu.cn/trRosetta/) were used as ab initio modeling methods to build models SCD_RB, SCD_CQ, and SCD_trR, respectively. The MpFADS6 model was constructed using trRosetta. The subsequent mutation models were based on the MpFADS6 model, built and optimized for energy by Chimera.

\section{Model evaluation}

Structural models were evaluated using QMEAN (Benkert et al.

2011) (https://swissmodel.expasy.org/qmean/) and SAVES (Laskowski et al.

1993) (https://servicesn.mbi.ucla.edu/SAVES/). In addition, to assess whether these modeling methods are suitable for fatty acid desaturases, two important indices were introduced to evaluate model accuracy (Martí-Renom et al. 2000): the degree of conservation (RMSD of conserved residues) and the correctness of alignment (TM score and global RMSD) between the target and template in Table 1. Using mTM-align (Dong et al. 2018) (https://yanglab.nankai.edu.cn/mTM-align/) to align all models of SCD1 and the crystal structure obtained by X-ray diffraction at a resolution of $2.60 \AA$, the TM score and 
structure-based phylogenetic tree were generated. The RMSD of the SA-CoA binding pocket in SCD1 is also an important indicator calculated using PyMOL (version 2.3.4).

\section{Molecular docking}

Based on the classification of released hotspot prediction servers, the FTMap (Kozakov et al. 2015) (https://ftmap.bu.edu/home.php) were used to predict the potential binding sites.

The docking procedures with the specific parameters are listed as follows:

For AutoDock Vina (version 4.2.6) (Morris et al. 2009)

AutoDockTools were used to convert the ligand and receptor files into the pdbqt format and specify them as ligand and macromolecule, respectively. The docking box was set, the configuration file was constructed, and AutoDock Vina was run.

For LeDock (version 1.0) (Zhang and Zhao 2016)

The command lepro was used to process the receptor protein and set the docking box. The docking program was run using the command ledock dock in.

For DINC (http://dinc.kavrakilab.org/) (Antunes et al. 2017)

The online docking service requires an input receptor structure file, ligand structure file, and corresponding parameters.

In these three programs, the docking box was determined using the spatial range formed by histidine conserved regions in FADS6, the protein-ligand binding hotspots to predict the spatial range of services, and the cavity of the protein structure.

For rdock, dock6, and Rosetta, the docking program was run according to the respective manuals. The docking box was determined by the program.

All RMSD values were calculated using PyMOL.

\section{Alanine substitution}

Twelve mutants of the MpFADS6 were constructed using site-directed mutagenesis. The corresponding primers for each mutant were synthesized by BGI Genomics (Shenzhen, China) and are listed in Table S1. The pYES2-MpFADS6 plasmid was used as the template. PCR was performed in a total volume of $50 \mu \mathrm{L}$ containing $5 \mu \mathrm{M}$ dNTP, $5 \mu \mathrm{M} 10 \times$ buffer, $2 \mu \mathrm{M} \mathrm{Mg}^{2+}, 0.5 \mu \mathrm{M}$ of each primer, $5 \mathrm{ng}$ of template plasmid DNA, and $1 \mathrm{U}$ of KOD DNA polymerase. Cycling conditions involved an initial denaturation at $95^{\circ} \mathrm{C}$ for 10 min, followed by 28 cycles of denaturation at $95^{\circ} \mathrm{C}$ for $30 \mathrm{~s}$, annealing at a temperature gradient (58- 
$74^{\circ} \mathrm{C}$ according to primers) for $30 \mathrm{~s}$, extension at $68^{\circ} \mathrm{C}$ for $5 \mathrm{~min}$, and a final extension at $68^{\circ} \mathrm{C}$ for $10 \mathrm{~min}$. Amplification quality was controlled by electrophoresis. The amplicon was digested with $\mathrm{Dpn} /$ at $37^{\circ} \mathrm{C}$ for $1 \mathrm{~h}$ to exclude parental non-mutated methylated plasmid DNA and transformed into competent $E$. coli DH5a cells. Plasmid from transformants was sequenced to confirm correct mutation (BGI Genomics).

\section{Protein expression and whole-cell biotransformation}

S. cerevisiae INVSc1, a strain that does not contain fatty acids C18:2 and C18:3, was transformed with the pYES2-MpFADS6 wild-type and mutant vectors. After culturing for $24 \mathrm{~h}$ at $28^{\circ} \mathrm{C}, 2 \%$ galactose was added to induce protein expression. After $24 \mathrm{~h}$, the cells were treated with exogenous fatty acid substrates $(0.25 \mathrm{mM} \mathrm{ALA})$ for $12 \mathrm{~h}$ at $28^{\circ} \mathrm{C}$ and harvested by centrifugation.

\section{Chromatographic analyses}

The method was modified and optimized according to a previously described method (Shi et al. 2015). The induced strains were lyophilized, and $1 \mathrm{~mL}$ of hydrochloric acid methanol and $20 \mu \mathrm{g}$ pentadecanoic acid was added to $50 \mathrm{mg}$ of freeze-dried cells. After methyl esterification at $70^{\circ} \mathrm{C}$ for $3 \mathrm{~h}, 1 \mathrm{~mL}$ of saturated sodium chloride was added, shaken, and mixed. Then, $1 \mathrm{~mL}$ of $\mathrm{n}$-hexane was added to extract fatty acid methyl esters, and the upper $n$-hexane layer was extracted by centrifugation at $2000 \times g$ for 5 min. Fatty acid methyl esters were then analyzed by gas chromatography-mass spectrometry (GC-MS) as previously described (Zhang et al. 2012).

\section{Statistical analysis}

Desaturase activity is represented by the conversion rate of ALA to $\mathrm{SDA}_{\mathrm{CR}} \mathrm{CR}_{\mathrm{ALA}}=(\mathrm{SDA}) /((\mathrm{ALA})+(\mathrm{SDA})) \times$ $100 \%$. All experiments were set up in three groups, and all data were statistically analyzed using SPSS Statistics 20 software (IBM, Armonk, NY, USA). The results are presented as mean \pm standard deviation, with significance indicated at $a<0.05$.

\section{Results}

\section{Comparison and construction of membrane-bound fatty acid desaturase model}

As the only active fatty acid desaturase complex crystal structure, the muSCD1_SA-CoA complex was parsed by X-ray diffraction. MpFADS6 is a membrane-bound desaturase that displays $<20 \%$ identity with proteins in the PDB and only $14.9 \%$ identity with muSCD1 (Figure S1). Therefore, it is necessary to compare the models constructed using different modeling programs to obtain a highly accurate fatty acid desaturase model. 
The SCD_SW homology model was constructed using the Swiss-model, which used the template manual screened in PDB. Models SCD_Cl, SCD_CA, SCD_RB, SCD_CQ, and SCD_trR were auto-generated by C-ITASSE, CATHER, Robetta, C-QUARK, and trRosetta, respectively. The global RMSD between the crystal and the five candidate models built by each program is shown in Fig. 1A. The model SCD_Cl was closest to the crystal. However, this method relies on multiple templates or protein fragments to construct the final model and the 4YMK template was used to construct the SCD_Cl. To fit FADS6, the following comparison excluded models that used 4YMK as a template. SCD_RB and SCD_trR were closer to muSCD1 in Fig. $1 \mathrm{~A}$. The TM-scores of both were $>0.5$, indicative of similarity with the crystal (Table 1 ). Alignment of muSCD1, SCD_RB, and SCD_trR (Fig. 1B) indicated that the RMSD of the transmembrane domains of these three models was $<1 \AA$, indicating that they were structurally conserved regions. The conformational consistency of these domains was higher than that of the cytoplasmic region interacting with the substrate. Although the global RMSD of SCD_trR reached $3.04 \AA$, the TM score showed that SCD_trR was highly similar to muSCD1. RMSD is the average distance between atoms of the system, and it seems that the loose regions in the overall protein, especially the $\mathrm{N}$ - or $\mathrm{C}$-terminus, increased the global RMSD value

To explore whether SCD_RB and SCD_trR can reflect the actual structure, SA-CoA was imported into the model and the distance between the interaction sites was analyzed. In Figure S2, We compared the changes in the spatial position of the residues that interact with the substrate SA-CoA which have been reported (Bai et al. 2015). It shows that the RMSD of the substrate tunnel between SCD_trR and SA-CoA was $<1 \AA$, compared to $3.8 \AA$ for the SCD_RB model (Table S2). In contrast, the distance between the residues of the SCD_trR model and the crystal was lower (Table S2). Although there were still some differences with muSCD1, the total deviation of the residues binding to the SA-CoA head group in SCD_trR was in the range of $2 \AA$ (Table S2). Overall, the $a b$ initio modeling method was more amenable for fatty acid desaturase than the other methods. Among the ab initio methods, the best model was constructed using trRosetta.

Therefore, the MpFADS6 model (Fig. 2) was constructed using trRosetta and the quality was evaluated using QMEAN and PROCKER. As shown in Table S3, 99.0\% and 100\% residues of the MpFADS6 model were in the most favored and additional allowed regions, respectively (Figure S3). QMEAN scores were accepted as -3.87. It indicated that the MpFADS6 model constructed by trRosetta, as well as SCD1_trR, were closest to the real confirmation and displayed satisfactory quality. Therefore, the MpFADS6 model was able to explore substrate binding sites.

\section{Docking to explore potential binding sites of delta 6 fatty acid desaturase with the substrate}

The MpFADS6 model in the present study had satisfactory stereochemical quality and was used for the docking of the substrate. However, the ligand head group of fatty acyl-CoA with higher flexibility caused the difficulty of docking to fatty acid desaturase. After evaluating the five mainstream academic docking procedures, it was found the best pose was obtained by dock6 in which the acyl chain extended well into 
the hydrophobic cavity in a reasonable range and formed a stable state with the key binding residues of fatty acid desaturase (Fig. 2 and Table 2). And LeDock and rDock showed better performance on ligand heme with few rotatable bonds (Fig. 4A and Table 2).

The acyl tail of ALA-COA was in a negatively charged cavity surrounded by three histidine conserved regions (Fig. 2B). The head group of CoA was attracted by the positive charge and anchored to the protein surface (Fig. 2C). As previously reported, heme bonded to the HPGG domain at the N-terminus of MpFADS6, in which a potential interaction site revealed that heme formed a П-П stack with residues $\mathrm{H} 69$ and H94 (Fig. 4A). Fig. 2B shows the potential sites where ALA-CoA interacted in the histidine conserved domains to introduce a double bond at the sixth position (highlighted in light red) from the methyl terminal. Residues $\mathrm{H} 231, \mathrm{H} 358, \mathrm{~K} 448$, and N445 may promote substrate stability. In addition, as a preferred substrate for MpFADS6, ALA is formed by dehydrogenation of LA at the fifteenth carbon atom from the methyl terminal via omega-3 desaturase. This means that the LA and ALA structures only differ by one double bond (highlighted in magenta in Fig. 2B. Residues H228, F352, W224, M227, and F289 might strengthen the desaturation of the interaction between the double bond of ALA and these residues, resulting in substrate preference.

\section{Effect of mutation site on substrate binding ability}

To confirm the influence of the amino acid sites analyzed above on desaturation, the 10 residues were mutated to alanine, and the corresponding Saccharomyces cerevisiae recombinants were constructed because $S$. cerevisiae does not contain ALA and SDA, which was the substrate and product of FADS6, respectively. After induction by galactose for $24 \mathrm{~h}$, ALA was added as a substrate for the enzymatic reaction, and the fatty acids were extracted for methyl esterification and analysis by GC-MS. The desaturase activity was shown in Fig. 3 and Table S4. The corresponding mutant models were built by Chimera and compared the structural difference with original model. And the affinities of the mutants to the substrate were analyzed by docking (Table 2). In addition to these 10 mutations by calculated analyses, two random mutations, S97A and M223A, were simultaneously analyzed for structural differences and enzyme activities.

As shown in Fig. 4A, $\mathrm{H} 94$ and $\mathrm{H} 69$ in the heme-binding region have the imidazole groups that attracted divalent iron at the heme center to form a stable binding. One of $\mathrm{H} 94$ and $\mathrm{H} 69$ was mutated to alanine, which suddenly lost the binding force and blocked desaturation (Fig. 3). The docking results in Table 2 show that H69A and H94A have low affinity for Heme, but the docking posts in Fig. 4B and C show that heme cannot form a stable binding with HPGG domain. However, the affinity of the random mutation S97A at the N-terminal reduced and Heme is right in the HPGG domain like Fig. 4A, so the deaturation activity retained $84.44 \%$ (Fig. 3). Therefore, H69A and H94A could not convert ALA into SDA, where electron transfer was most likely blocked because of the unstable reaction to heme.

In addition, one mutant among W290A, W224A, and F352A directly bound the substrate, which led to the inactivation of MpFADS6 (Fig. 3). According to the substrate mesh (Fig. 5), one of the mutants rendered 
the residues in the substrate tunnel incapable of contacting the substrate acyl chain, which failed to recognize the double bond of ALA-CoA and switch on desaturation. In contrast, the side chain group of F289A was distant from the substrate, which made the cavity space larger (Fig. 5E). This weakened the stability of the complex state of the protein and the substrate. The activity and affinity of MpFADS6 decreased, but inactivation was not observed after the mutation of F289, in which the deaturation activity retained $85.72 \%$ (Table 2 and Fig. 3). However, the random mutation M223A has $99.95 \%$ activity of MpFADS6 (Fig. 3). Molecular simulation analysis indicated that M223 was far from the cavity of the protein substrate that was not in contact with the substrate (Figure S4). The conformation of W224 and W290, which constituted the substrate channel, remained unchanged during the methionine to alanine mutation. Therefore, M223A was not the key binding residue of MpFADS6.

The head group of the ALA-COA substrate was located on the surface of the protein and had pronounced flexibility. Many residues contacted the MpFADS6 surface, which made the analysis difficult. Therefore, $\mathrm{H} 452$, N445, K448, and H358 residues located at the entrance of the substrate cavity were selected for investigation. Alanine mutations were detected on these four amino acids (Fig. 6). The increased activity of K448A was speculated to reflect the enlarged entrance of the cavity after mutation (Fig. 6B), which increased the recognition and attraction of MpFADS6 to the substrate and blocked the electrostatic attraction of lysine to the CoA group. These changes increased the probability that the substrate would enter the cavity in the correct posture. Therefore, the desaturation activity of K448A increased by 1.08 times (Fig. 3). Due to the electrostatic interaction, N445A lost its covalent connection with the adenosine group (Fig. 6C). After mutations of $\mathrm{H} 452$ and $\mathrm{H} 358$, the positively charged domains were converted to neutral (Fig. 6D and E), which weakened the protein surface and attracted the adenosine group of ALACoA. Thereby reducing the recognition and attraction of MpFADS6 to the substrate, resulting in a significant decrease in the activity, and the retained activities are less than $50 \%$ of wild type (Fig. 3 ).

\section{Discussion}

Fatty acid desaturase is composed of multiple transmembrane domains that are difficult to express and purify. The lack of structural information and the low sequence identity with other proteins make it difficult to obtain insights into the desaturation mechanism from sequence information. To date, the only active fatty acid desaturase complex crystal structure, muSCD1, was parsed by X-ray diffraction which was used in the present study to evaluate the current modeling and docking programs.

Generally, the sequence identity of proteins with different functions in the fatty acid desaturase family is $<30 \%$, and the quality of the models constructed by homology modeling lacks confidence. As the representatives of the threading approach, C-I-TASSER and CATHER are based on multiple templates or protein fragments to construct the final model, which has been suggested to be more accurate than a single template. CASP showed that comparative modeling based on multiple templates or protein fragment recombination to construct a model has more advantages than a single template (Venclovas and Margelevicius 2005). However, in terms of membrane-bound desaturase modeling, the credibility of the models SCD_Cl and SCD_CA greatly depend on the template 4YMK. In contrast, the ab initio modeling 
method is more suitable for fatty acid desaturases with no aligned templates to build a high-quality model. The SCD1_trR and MpFADS6 models constructed by trRosetta were closest to the real confirmation and displayed satisfactory quality. The models still had defects. The local entropy accumulation, bond angle, and bond length affected the total score. However, $95 \%$ of the residues in the Ramachandran diagram remained within the allowable range, indicating their high quality. Therefore, the MpFADS6 model was able to explore substrate binding sites. With the announcement of Alphafold, the Alphafold Protein Structure Database (https://www.alphafold.ebi.ac.uk/) shows mammalian FADS6 models from four species, Danio rerio FADS6, Homo sapiens FADS6, Mus musculus FADS6, Rattus norvegicus FADS6. Comparing the MpFADS6 model constructed by trRosetta with these mammalian FADS6 models (Table S5), the results show that the global RMSD between the MpFADS6 model and the four mammalian FADS6 models is about $4.369 \AA$, while the average RMSD of the conserved domain is $<1.0 \AA$, while the sequence identities are $<25 \%$. It suggests that the FADS 6 model constructed by trRosetta is highly consistent with the model constructed by Alphafold and these methods are excellent for constructing structural models of fatty acid desaturases.

The substrate of the fatty acid desaturase is in the form of fatty acyl-CoA with about more than forty rotatable atoms and a flexible conformation to enhance the difficulty of the best pose. The manner in which the entire protein is used as the target range during docking weakens the calculation speed and increases the analysis because of redundant results. If the spatial range of interaction can be determined beforehand, these defects would be reduced. Based on geometric or energy features, FTMap was able to reveal partially enclosed cavities or shallow cavities by detecting the energy potential between the probes and the cavity, providing accurate identification of binding sites by manually analyzing the interaction between each probe and its surrounding residues. Based on reliable protein structure and reasonable and accurate docking range, the six docking procedures used in this work positioned the substrate acyl-CoA into the cavity of desaturases. The partial output poses featured excessively large-amplitude torsion, which did not interact with the residues in the substrate tunnel due to the plasticity of the long-chain substrate. Most docking poses outputted by dock6 and Rosetta entered the substrate tunnel in the correct direction and displayed stretched states. However, the flexible head group of the substrate displayed an increased RMSD with the original conformation. The heme docking molecule has a small number of rotary atoms. Autodock Suite, LeDock, and rdock provided consistent poses. These programs are convenient, fast, and accurate.

The current researches on the interaction between fatty acid desaturases and substrates and the substrate specificities of fatty acid desaturases focused on using sequence alignment combined with site-directed mutagenesis or fragment exchange technology ( Li et al. 2020; Rong et al. 2019a; Rong et al. 2019b; Shi et al. 2015; Shi et al. 2018). In this work, the potential substrate interaction sites of MpFADS6 were determined by docking and confirmed by site-directed mutagenesis. The changed conformation of the protein was analyzed by amino acid mutations in molecular simulations to illustrate the molecular mechanism of desaturation. By replacing the amino acid with alanine to substitute the active group on the side chain with a small-volume methyl group, which has only a small effect on the structure of the protein, the influence of the residue on the enzyme activity could be explored. It is necessary to analyze 
the nature of the amino acids around the mutated residue and the interaction between the mutated residue and the ligand. Some amino acid residues play a key role in the interaction with the ligand.

This study provides new insights and directions for subsequent work. However, there are still some limitations, such as whether the stability of fatty acid desaturase conformation changes after mutations or anchors the phospholipid bilayer. These aspects need to be further explored in combination with molecular dynamics simulations.

\section{Abbreviations}

PUFA, polyunsaturated fatty acid, FADS6, delta 6 fatty acid desaturase, LA, linoleic acid, GLA, gammalinolenic acid, ALA, linoleic acid, SDA, stearidonic acid, PDB, Protein Data Bank, SA-CoA, stearoyl-CoA, ALA-CoA, linoleinyl-CoA, GC-MS, gas chromatography-mass spectrometry, 3D, three-dimensional.

\section{Declarations}

\section{Ethics approval and consent to participate}

Not applicable

\section{Consent for publication}

Not applicable

\section{Competing interests}

Not applicable

\section{Availability of data and material}

The authors declare that the data supporting the findings of this study are available within the article and its supplementary information files.

\section{Funding}

This research was funded by the National Key Research and Development Program of China (2021YFC2100700), the National Natural Science Foundation of China (grant number 31722041), and the program of 'Collaborative innovation center of food safety and quality control in Jiangsu Province'.

\section{Authors' Contributions}

All authors have read and agree to the published version of the manuscript. Conceptualization, J.C. and H.C., methodology, J.C and H.C., software, J.C., validation, J.C. and H.C., formal analysis, J.C. and H.C., 
resources, H.C., X.T., H.Z., Y.C., and W.C., writing-original draft preparation, J.C., writing-review and editing, H.C., visualization, J.C., funding acquisition, H.C., X.T., Z.G., H.Z., Y.C. and W.C.

\section{Acknowledgements}

We are also grateful to the Meiler Lab, Vanderbilt University, for sharing the video lectures and tutorials about Rosetta.

\section{Author information}

\section{Affiliations}

State Key Laboratory of Food Science and Technology, School of Food Science and Technology, Jiangnan University, Wuxi 214122, People's Republic of China

Jie Cui, Haiqin Chen, Xin Tang, Hao Zhang, Yong Q. Chen and Wei Chen

School of Food Science and Technology, Jiangnan University, Wuxi 214122, P. R. China

Jie Cui, Haiqin Chen, Xin Tang, Hao Zhang, Yong Q. Chen and Wei Chen

National Engineering Research Center for Functional Food, Jiangnan University, Wuxi 214122, P. R. China

Hao Zhang and Wei Chen

Wuxi Translational Medicine Research Center and Jiangsu Translational Medicine Research Institute Wuxi Branch, Wuxi 214122, P. R. China

Hao Zhang

Department of Cancer Biology, Wake Forest School of Medicine, Winston-Salem, NC 5: 27127, USA

Yong Q. Chen

\section{Corresponding author}

Correspondence to Haiqin Chen.

\section{References}

1. Alford RF, Koehler Leman J, Weitzner BD, Duran AM, Tilley DC, Elazar A, Gray JJ (2015) An Integrated Framework Advancing Membrane Protein Modeling and Design. PLoS Comput Biol 11(9):e1004398 https://doi.org/10.1371/journal.pcbi.1004398 
2. Altschul SF, Gish W, Miller W, Myers EW, Lipman DJ (1990) Basic local alignment search tool. J Mol Bio 215(3):403-410 https://doi.org/10.1016/s0022-2836(05)80360-2

3. Antunes DA, Moll M, Devaurs D, Jackson KR, Lizée G, Kavraki LE (2017) DINC 2.0: A New ProteinPeptide Docking Webserver Using an Incremental Approach. Cancer Res 77(21):e55-e57 https://doi.org/10.1158/0008-5472.Can-17-0511

4. Bai Y, McCoy J, Levin E, Zhou M (2015) X-Ray Structure of a Mammalian Stearoyl-Coa Desaturase-1. Biophys J 108:534a https://doi.org/10.1016/j.bpj.2014.11.2926

5. Benkert P, Biasini M, Schwede T (2011) Toward the estimation of the absolute quality of individual protein structure models. Bioinformatics (Oxford, England) 27(3):343-350 https://doi.org/10.1093/bioinformatics/btq662

6. Chen KY, Sun J, Salvo JS, Baker D, Barth P (2014) High-resolution modeling of transmembrane helical protein structures from distant homologues. PLoS Comput Biol 10(5):e1003636 https://doi.org/10.1371/journal.pcbi.1003636

7. Dong R, Pan S, Peng Z, Zhang Y, Yang J (2018) MTM-align: A server for fast protein structure database search and multiple protein structure alignment. Nucleic Acids Res 46(W1):w380-w386 https://doi.org/10.1093/nar/gky430

8. Dowhan W, Vitrac H, Bogdanov M (2019) Lipid-Assisted Membrane Protein Folding and Topogenesis. Protein J 38(3):274-288 https://doi.org/10.1007/s10930-019-09826-7

9. Du Z, Pan S, Wu Q, Peng Z, Yang J (2020) CATHER: a novel threading algorithm with predicted contacts. Bioinformatics (Oxford, England) 36(7):2119-2125 https://doi.org/10.1093/bioinformatics/btz876

10. Forli S, Huey R, Pique ME, Sanner MF, Goodsell DS, Olson AJ (2016) Computational protein-ligand docking and virtual drug screening with the AutoDock suite. Nat Protoc 11(5):905-919 https://doi.org/10.1038/nprot.2016.051

11. Kozakov D, Grove LE, Hall DR, Bohnuud T, Mottarella SE, Luo L, Xia B, Beglov D, Vajda S (2015) The FTMap family of web servers for determining and characterizing ligand-binding hot spots of proteins. Nat Protoc 10(5):733-755 https://doi.org/10.1038/nprot.2015.043

12. Laskowski RA, MacArthur MW, Moss DS, Thornton JM (1993) PROCHECK: a program to check the stereochemical quality of protein structures. J Appl Crystallogr 26(2):283-291 https://doi.org/10.1107/s0021889892009944

13. Li D, Damry AM, Petrie JR, Vanhercke T, Singh SP, Jackson CJ (2020) Consensus Mutagenesis and Ancestral Reconstruction Provide Insight into the Substrate Specificity and Evolution of the Front-End $\Delta 6$-Desaturase Family. Biochemistry 59(14):1398-1409 https://doi.org/10.1021/acs.biochem.0c00110

14. Madeira F, Park YM, Lee J, Buso N, Gur T, Madhusoodanan N, Basutkar P, Tivey ARN, Potter SC, Finn RD, Lopez R (2019) The EMBL-EBI search and sequence analysis tools APIs in 2019. Nucleic Acids Res 47(W1):w636-w641 https://doi.org/10.1093/nar/gkz268 
15. Martí-Renom MA, Stuart AC, Fiser A, Sánchez R, Melo F, Sali A (2000) Comparative protein structure modeling of genes and genomes. Annu Rev Bioph Biom 29:291-325 https://doi.org/10.1146/annurev.biophys.29.1.291

16. Melcr J, Ferreira TM, Jungwirth P, Ollila OHS (2020) Improved Cation Binding to Lipid Bilayers with Negatively Charged POPS by Effective Inclusion of Electronic Polarization. J Chem Theory Comput 16(1):738-748 https://doi.org/10.1021/acs.jctc.9b00824

17. Morris GM, Huey R, Lindstrom W, Sanner MF, Belew RK, Goodsell DS, Olson AJ (2009) AutoDock4 and AutoDockTools4: Automated docking with selective receptor flexibility. J Comput Chem 30(16):2785-2791 https://doi.org/10.1002/jcc.21256

18. Rong C, Chen H, Tang X, Gu Z, Zhao J, Zhang H, Chen W, Chen Y (2019a) Characterization and molecular docking of new $\Delta 17$ fatty acid desaturase genes from Rhizophagus irregularis and Octopus bimaculoides. RSC Advances 9:6871-6880 https://doi.org/10.1039/C9RA00535H

19. Rong C, Chen H, Tang X, Gu Z, Zhao J, Zhang H, Chen Y, Chen W (2019b) Structural Determinants of Substrate Specificity of Omega-3 Desaturases from Mortierella alpina and Rhizophagus irregularis by Domain-Swapping and Molecular Docking. Int J Mol Sci 20(7):1603 https://doi.org/10.3390/ijms20071603

20. Shen J, Wu G, Tsai AL, Zhou M (2020) Structure and Mechanism of a Unique Diiron Center in Mammalian Stearoyl-CoA Desaturase. J Mol Bio 432(18):5152-5161 https://doi.org/10.1016/j.jmb.2020.05.017

21. Shi H, Chen H, Gu Z, Song Y, Zhang H, Chen W, Chen YQ (2015) Molecular mechanism of substrate specificity for delta 6 desaturase from Mortierella alpina and Micromonas pusilla. J Lipid Res 56(12):2309-2321 https://doi.org/10.1194/jlr.M062158

22. Shi H, Wu R, Zheng Y, Yue X (2018) Molecular mechanisms underlying catalytic activity of delta 6 desaturase from Glossomastix chrysoplasta and Thalassiosira pseudonana. J Lipid Res 59(1):79-88 https://doi.org/10.1194/jlr.M079806

23. Tosi F, Sartori F, Guarini P, Olivieri O, Martinelli N (2014) Delta-5 and delta-6 desaturases: crucial enzymes in polyunsaturated fatty acid-related pathways with pleiotropic influences in health and disease. Adv Exp Med Biol 824:61-81 https://doi.org/10.1007/978-3-319-07320-0_7

24. Venclovas C, Margelevicius M (2005) Comparative modeling in CASP6 using consensus approach to template selection, sequence-structure alignment, and structure assessment. Proteins 61 Suppl 7:99105 https://doi.org/10.1002/prot.20725

25. Wang H, Klein MG, Zou H, Lane W, Snell G, Levin I, Li K, Sang BC (2015) Crystal structure of human stearoyl-coenzyme A desaturase in complex with substrate. Nat Struct Mol Biol 22(7):581-585 https://doi.org/10.1038/nsmb.3049

26. Wang J, Yazdani S, Han A, Schapira M (2020) Structure-based view of the druggable genome. Drug discov today 25(3):561-567 https://doi.org/10.1016/j.drudis.2020.02.006

27. Wang Y, Barth P (2015) Evolutionary-guided de novo structure prediction of self-associated transmembrane helical proteins with near-atomic accuracy. Nat Commun 6:7196 
https://doi.org/10.1038/ncomms8196

28. Wang Z, Sun H, Yao X, Li D, Xu L, Li Y, Tian S, Hou T (2016) Comprehensive evaluation of ten docking programs on a diverse set of protein-ligand complexes: the prediction accuracy of sampling power and scoring power. Phys Chem Chem Phys 18(18):12964-12975 https://doi.org/10.1039/c6cp01555g

29. Waterhouse A, Bertoni M, Bienert S, Studer G, Tauriello G, Gumienny R, Heer FT, de Beer TAP, Rempfer C, Bordoli L, Lepore R, Schwede T (2018) SWISS-MODEL: homology modelling of protein structures and complexes. Nucleic Acids Res 46(W1):w296-w303 https://doi.org/10.1093/nar/gky427

30. Yang J, Anishchenko I, Park H, Peng Z, Ovchinnikov S, Baker D (2020) Improved protein structure prediction using predicted interresidue orientations. P Nati Acad Sci U S A 117:201914677 https://doi.org/10.1073/pnas.1914677117

31. Yang J, Zhang Y (2015) I-TASSER server: new development for protein structure and function predictions. Nucleic Acids Res 43(W1):w174-w181 https://doi.org/10.1093/nar/gkv342

32. Zhang B, Rong C, Chen H, Song Y, Zhang H, Chen W (2012) De novo synthesis of trans -10 , cis-12 conjugated linoleic acid in oleaginous yeast Yarrowia lipolytica. Microb Cell Fact 11:51 https://doi.org/10.1186/1475-2859-11-51

33. Zhang N, Zhao H (2016) Enriching screening libraries with bioactive fragment space. Bioorg Med Chem Lett 26(15):3594-3597 https://doi.org/10.1016/j.bmcl.2016.06.013

34. Zhao J, Cao Y, Zhang L (2020) Exploring the computational methods for protein-ligand binding site prediction. Comput Struct Biotechnol J 18:417-426 https://doi.org/10.1016/j.csbj.2020.02.008

35. Zheng W, Li Y, Zhang C, Pearce R, Mortuza SM, Zhang Y (2019) Deep-learning contact-map guided protein structure prediction in CASP13. Proteins 87(12):1149-1164 https://doi.org/10.1002/prot.25792

\section{Tables}

Table 1. Evaluation of the overall difference between the constructed model and the crystal structure of muSCD1. 


\begin{tabular}{lllll} 
& TM-score $^{1}$ & Global RMSD & RMSD of tunnel & \\
& & & Model method \\
\hline muSCD1 & 1.0000 & 0.0000 & 0.0000 & PDB ID 4YMK \\
\hline SCD_Cl & 0.9958 & 0.4470 & - & C-I-TASSER \\
\hline SCD_trR & 0.8296 & 3.0400 & 0.7240 & trRosetta \\
\hline SCD_RB & 0.6478 & 0.4980 & 3.8030 & Robetta \\
\hline SCD_CQ & 0.4148 & 4.5180 & - & C-QUARK \\
\hline SCD_SW & 0.3342 & 4.7420 & - & Swiss-model \\
\hline SCD_CA & 0.2798 & 6.6120 & - & CATHER
\end{tabular}

1 TM Score is an index used to detect the structural similarity between two proteins. The TM Score is between 0 and 1 , where 1 indicates that the two are completely consistent.

2 The global RMSD value measures the degree of difference between the two structures.

3 The RMSD of the residues composed of the substrate tunnel indicated the difference between model and crystal. The amino acid selected here is consistent with Fig. 4D.

"-" means this model was not compared.

Table 2. Docking scores of MpFADS6 mutants with ALA-CoA. 


\begin{tabular}{|c|c|c|c|c|}
\hline Residue position & Mutants & Heme ${ }^{1}$ & ALA-CoA ${ }^{2}$ & $\mathrm{RMSD}^{3}$ \\
\hline & WT & -4.5 & -6.01 & 0.000 \\
\hline \multirow[t]{3}{*}{$\mathrm{N}$-terminal } & H69A & -4.9 & & 0.140 \\
\hline & H94A & 0.0 & & 0.140 \\
\hline & S97A & -3.9 & & 0.140 \\
\hline \multirow[t]{5}{*}{ Substrate tunnel } & W224A & & - & 0.000 \\
\hline & W290A & & - & 0.000 \\
\hline & F352A & & -5.44 & 0.000 \\
\hline & F289A & & -4.99 & 0.000 \\
\hline & M223A & & -5.78 & 0.000 \\
\hline \multirow[t]{4}{*}{ Surface } & $\mathrm{K} 448 \mathrm{~A}$ & & -6.44 & 0.000 \\
\hline & H358A & & -4.02 & 0.000 \\
\hline & N445A & & -4.52 & 0.000 \\
\hline & $\mathrm{H} 452 \mathrm{~A}$ & & -5.65 & 0.000 \\
\hline
\end{tabular}

1 Affinity for the mutants with the substrate Heme by Ledock $(\mathrm{kcal} / \mathrm{mol})$.

2 Affinity for the mutants with the substrate ALA-CoA by Dock6 $(\mathrm{kcal} / \mathrm{mol})$.

3 The global RMSD of alignment of model MpFADS6 and mutant.

"-" means the substrate is not bound to the mutant.

\section{Figures}

\section{Figure 1}

Comparison between the structure of SCD1 and models constructed using different modeling methods. (A). The average global RMSD between the models was constructed using the six modeling methods and the data from the crystal. (B). Alignment of SCD1 and models. The models with a global RMSD $<2 \AA$ compared with SCD1. The purple cartoon is a common core region with three structures. 


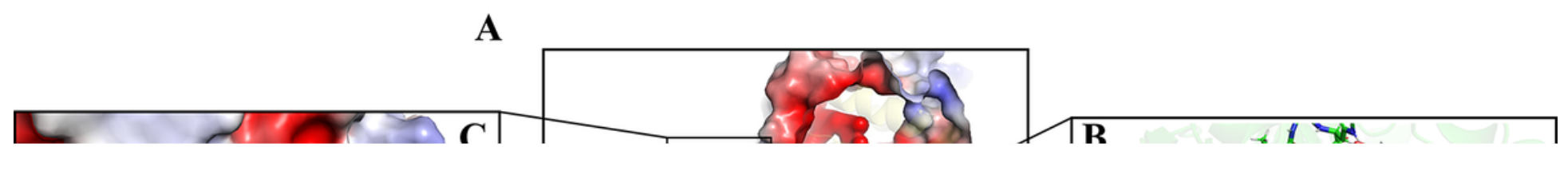

Figure 2

Molecular docking simulation showing the potential interaction of MpFADS6 with ALA-CoA. (A).

Electrostatic surface of MpFADS6_ALA-CoA complex (B). Cartoon of the MpFADS6_ALA-CoA complex. (C). Electrostatic surface of the head group in the MpFADS6_ALA-CoA complex. The atoms are highlighted in light red in ALA-CoA, indicating the sixth and seventh carbons from the methyl end. The atoms highlighted in magenta indicate the position of the fatty acid unsaturated bond.

\section{Figure 3}

Activities of wild-type MpFADS6 and mutants to ALA. The sites predicted to constitute the substrate channel of MpFADS6 are indicated in red. The sites of MpFADS6 predicted to bind heme are indicated in light blue. The sites of MpFADS6 predicted to recognize and bind the head group of ALA-CoA is indicated in blue. Mutant obtained by accident is indicated in yellow. ${ }^{*}, p<0.05 . * *, p<0.01 . * \star *, p<0.001$.

\section{Figure 4}

Key binding sites of MpFADS6 to heme (A). Conformational changes in MpFADS6 due to mutations H94A (B) and H69A (C). Yellow stick indicates heme with divalent iron in the center. The salmon stick indicates the Cyt b5-like domain at the N-terminus of MpFADS6.

\section{Figure 5}

The key amino acids in the substrate cavity of MpFADS6 (A). Conformational changes in MpFADS6 due to mutations W224 (B), W290 (C), F352A (D), and F289A (E). The yellow stick indicates the mesh of substrate ALA-COA. The red atom represents the position of the double bond induced by FADS6. The magenta atom represents the double bond at C15 of ALA.

\section{Figure 6}


The key amino acid site for MpFADS6 binding to the substrate head group (A). Conformational changes in K448A (B), N445A (C), N452A (D), and H358A (E). The yellow stick indicates the mesh of substrate ALA-COA.

\section{Supplementary Files}

This is a list of supplementary files associated with this preprint. Click to download.

- GA.tif

- Supplementarymaterials.pdf 